\title{
Standardized assessment of the signal intensity increase on unenhanced T1-weighted images in the brain: the European Gadolinium Retention Evaluation Consortium (GREC) Task Force position statement
}

\author{
Carlo C. Quattrocchi ${ }^{1}$ (D) Joana Ramalho ${ }^{2}$ - Aart J. van der Molen ${ }^{3}$ Àlex Rovira ${ }^{4}$. Alexander Radbruch ${ }^{5}$ on behalf of \\ the GREC, European Gadolinium Retention Evaluation Consortium and the ESNR, European Society of Neuroradiology
}

Received: 9 June 2018 / Revised: 29 August 2018 / Accepted: 25 September 2018 / Published online: 9 November 2018

(C) European Society of Radiology 2018

\begin{abstract}
After the initial report in 2014 on T1-weighted (T1w) hyperintensity of deep brain nuclei following serial injections of linear gadolinium-based contrast agents (GBCAs), a multitude of studies on the potential of the marketed GBCAs to cause T1w hyperintensity in the brain have been published. The vast majority of these studies found a signal intensity (SI) increase for linear GBCAs in the brain - first and foremost in the dentate nucleus - while no SI increase was found for macrocyclic GBCAs. However, the scientific debate about this finding is kept alive by the fact that SI differences do not unequivocally represent the amount of gadolinium retained. Since the study design of the SI measurement in various brain structures is relatively simple, MRI studies investigating gadolinium-dependent T1w hyperintensity are currently conducted at multiple institutions worldwide. However, methodological mistakes may result in flawed conclusions. In this position statement, we assess the methodological basis of the published retrospective studies and define quality standards for future studies to give guidance to the scientific community and to help identify studies with potentially flawed methodology and misleading results.

\section{Key Points}

- A multitude of studies has been published on the potential of the marketed GBCAs to cause T1w hyperintensity in the brain.

- The gadolinium-dependent T1w hyperintensity in the brain depends on patient's history, types of GBCAs used (i.e., linear vs. macrocyclic GBCAs) and MR imaging setup and protocols.

- Quality standards for the design of future studies are needed to standardize methodology and avoid potentially misleading results from retrospective studies.
\end{abstract}

Keywords Contrast media $\cdot$ Gadolinium $\cdot$ Magnetic resonance imaging $\cdot$ Dentate nucleus

Carlo C. Quattrocchi

c.quattrocchi@unicampus.it

1 Unit of Diagnostic Imaging and Interventional Radiology, Departmental Faculty of Medicine and Surgery, Università Campus Bio-Medico di Roma, via Alvaro del Portillo 21, 00128 Rome, Italy

2 Department of Neuroradiology, Centro Hospitalar de Lisboa Central, Alameda Santo António dos Capuchos, 1169-050 Lisbon, Portugal

3 Department of Radiology, C2-S, Leiden University Medical Center, Albinusdreef 2, NL-2333 ZA Leiden, The Netherlands

4 Section of Neuroradiology Vall d'Hebron University Hospital, Passeig de la Vall d'Hebron, 119-129, 08035 Barcelona, Spain

5 Department of Radiology, German Cancer Research Center (DKFZ), Im Neuenheimer Feld 280, 69120 Heidelberg, Germany

$\begin{array}{ll}\text { Abbreviations } \\ \text { ACR } & \text { American College of Radiology } \\ \text { DN } & \text { Dentate nucleus } \\ \text { EMA } & \text { European Medicine Agency } \\ \text { ESUR } & \text { European Society of Urogenital Radiology } \\ \text { FDA } & \text { Food and Drug Administration } \\ \text { GBCA } & \text { Gadolinium-based contrast agent } \\ \text { GP } & \text { Globus pallidus } \\ \text { GREC } & \text { Gadolinium Retention Evaluation Consortium } \\ \text { NSF } & \text { Nephrogenic systemic fibrosis } \\ \text { SI } & \text { Signal intensity } \\ \text { T1w } & \text { T1-weighted }\end{array}$




\section{Background and framework}

In 2014, Kanda et al [1] reported a correlation between the number of gadolinium-based contrast agent (GBCA) injections and the increase of the dentate nucleus (DN)-to-pons and globus pallidus (GP)-to-thalamus signal intensity (SI) ratios on unenhanced T1-weighted (T1w) magnetic resonance imaging (MRI). Subsequent studies on human tissue found a positive correlation between the MRI signal changes and gadolinium concentration. Retrospective analysis at a multitude of sites revealed that gadolinium retention in brain structures in patients with normal renal function and intact blood-brain barrier has been occurring for the last 25 years without being noticed.

The majority of MRI studies [1-63] were focused on SI changes in the DN. Due to the relatively simple setup of such studies, retrospective imaging studies have been conducted worldwide at multiple sites. These studies provided evidence that (i) linear GBCAs are correlated with a SI increase in the DN if a certain number of injections (probably 5-6 injections) is exceeded; (ii) non-ionic linear GBCA gadodiamide causes a stronger SI increase than ionic linear GBCAs gadopentetate and gadobenate, and (iii) macrocyclic GBCAs are not or only weakly associated with SI increase compared to linear GBCAs (Fig. 1).

Notably, the majority of dentate studies found the SI increase to be dose-dependent for linear agents with a greater deposition associated especially with the weaker chelates such as gadodiamide (Omniscan ${ }^{\circledR}$, GE Healthcare) and gadopentetate dimeglumine (Magnevist $₫$, Bayer Healthcare) and in the presence of renal impairment. Indeed, the more stable macrocyclic GBCAs, gadoteridol (ProHance®), Bracco Imaging), gadoterate meglumine (Dotarem ${ }^{\circledR}$, Guerbet), and gadobutrol (Gadovist ${ }^{\circledR}$, Bayer Healthcare), have not been associated with substantial MRI changes in the majority of published studies, suggesting that the molecular structure of the GBCA ligand, whether linear or macrocyclic, is a crucial factor for the increase in signal intensity. When interpreting MRI studies, the inherent limitations of this technique to assess gadolinium in human tissues should be acknowledged.

A comparison between different imaging studies is often hampered by different methodological approaches, and stakeholders' interests can influence the debate by specifically presenting the publications that provide the desired results. The lack of systematic collection of data, the limited number of patients in single-center studies, and the varying technical setup and MRI sequence parameters among different institutions make data metaanalysis challenging if not impossible.

The current consensus paper aims to provide a guideline for retrospective MRI studies to guarantee a minimum level of standardization to achieve higher accuracy and maximize reproducibility. We highly urge editors and authors to comply with these standards to avoid the dilution of the scientific debate by flawed publications.
Fig. 1 Color map of currently published retrospective clinical studies on gadolinium-related brain T1w hyperintensity. Red: dentate nucleus (DN) signal increase with visible hyperintensity (unconfounded GBCAs); Light red: DN signal increase or visible hyperintensity (confounded GBCAs); Orange: DN T1w signal intensity increase without visible hyperintensity (unconfounded GBCAs); Light green: No DN signal increase or visible hyperintensity (confounded GBCAs); Green: No DN signal increase with no visible hyperintensity (unconfounded GBCAs)

\section{Scientific context}

In the period between 2014 and 2018, 63 original retrospective studies [1-63] were published all over the world, regarding the relationship between T1 high SI on brain MRI studies and exposure to intravenously injected GBCAs.

Available literature found that the signal changes in certain central nervous system structures identified with MRI, first and foremost the $\mathrm{DN}$, correlated positively with the exposure to linear GBCAs. It is under debate if macrocyclic GBCAs can cause any SI increase (Fig. 1).

Even though retrospective studies can be highly qualified, the level of evidence provided from any study reporting on $\mathrm{T} 1 \mathrm{w}$ hyperintensity depends primarily on uniform imaging protocol, scanners, time periods in which the follow-up examinations are performed and the number of included patients. Studies assessing identical imaging parameters and MR scanners at regular follow-up intervals provide a much higher level of evidence than studies that do not control for these parameters. Even though evidence from retrospective studies is generally classified as levels $3 \mathrm{~b}$ (individual case-control studies), 4 (case series), and 5 (expert opinion) according to the Centre for Evidence-Based Medicine, Oxford, they can therefore provide a higher level of evidence than prospective studies that do not control for these parameters. Given the current body of evidence, it is likely that prospective, blind, controlled randomized studies would not significantly change the consistent results of the numerous available retrospective studies on the potential of GBCAs to cause T1w hyperintensity. Nevertheless, type I (false positives findings) and type II errors (false negative findings) may flaw study results and data interpretation (Table 1). Editors need to evaluate methodology used in retrospective studies across the world due to the heavily increasing number of published studies on this topic.

Although no definitive symptoms or diseases linked to the $\mathrm{T} 1 \mathrm{w}$ hyperintensity in the gadolinium-exposed population have been reported, data on long-term effects is still limited, and further research is needed [64].

To date, nephrogenic systemic fibrosis (NSF) still represents the only well-established clinical entity related to toxic effects of gadolinium and occurs in patients with severe impairment of renal function after exposure to certain GBCAs.

Several papers have been published recently and presumably many will follow as radiological institutions are interested to 


\begin{tabular}{|c|c|c|c|c|c|c|c|c|}
\hline \multirow{2}{*}{$\begin{array}{l}\text { Chemical } \\
\text { Structure } \\
\text { Molecule }\end{array}$} & \multicolumn{2}{|c|}{$\begin{array}{c}\text { Linear } \\
\text { Non-Ionic }\end{array}$} & \multicolumn{3}{|c|}{$\begin{array}{c}\text { Linear } \\
\text { Ionic }\end{array}$} & \multicolumn{2}{|c|}{$\begin{array}{c}\text { Macrocyclic } \\
\text { Non-Ionic }\end{array}$} & \multirow{2}{*}{\begin{tabular}{|c|}
$\begin{array}{c}\text { Macrocyclic } \\
\text { Ionic }\end{array}$ \\
$\begin{array}{c}\text { gadoterate } \\
\text { meglumine }\end{array}$
\end{tabular}} \\
\hline & gadodiamide & gadoversetamid & $\begin{array}{l}\text { gadopentetate } \\
\text { dimeglumine }\end{array}$ & \begin{tabular}{|c|} 
gadobenate \\
dimeglumine
\end{tabular} & $\begin{array}{l}\text { gadoxetate } \\
\text { disodium }\end{array}$ & gadoteridol & gadobutrol & \\
\hline Marketed as & Omniscan & Optimark & Magnevist & MultiHance & Primovist & ProHance & Gadovist & Dotarem \\
\hline \multicolumn{9}{|l|}{$\# 1$} \\
\hline \multicolumn{9}{|l|}{$\# 2$} \\
\hline \multicolumn{9}{|l|}{$\# 3$} \\
\hline \multicolumn{9}{|l|}{$\# 4$} \\
\hline \multicolumn{9}{|l|}{$\# 5$} \\
\hline \multicolumn{9}{|l|}{$\# 6$} \\
\hline \multicolumn{9}{|l|}{$\# 7$} \\
\hline$\# 8$ & & & & & & & & \\
\hline$\# 9$ & & & & & & & & \\
\hline$\# 10$ & & & & & & & & \\
\hline$\# 11$ & & & & & & & & \\
\hline$\# 12$ & & & & & & & & \\
\hline$\# 13$ & & & & & & & & \\
\hline$\# 14$ & & & & & & & & \\
\hline$\# 15$ & & & & & & & & \\
\hline$\# 16$ & & & & & & & & \\
\hline$\# 17$ & & & & & & & & \\
\hline$\# 18$ & & & & & & & & \\
\hline$\# 19$ & & & & & & & & \\
\hline$\# 20$ & & & & & & & & \\
\hline$\# 21$ & & & & & & & & \\
\hline$\# 22$ & & & & & & & & \\
\hline$\# 23$ & & & & & & & & \\
\hline$\# 24$ & & & & & & & & \\
\hline$\# 25$ & & & & & & & & \\
\hline$\# 26$ & & & & & & & & \\
\hline$\# 27$ & & & & & & & & \\
\hline$\# 28$ & & & & & & & & \\
\hline$\# 29$ & & & & & & & & \\
\hline$\# 30$ & & & & & & & & \\
\hline$\# 31$ & & & & & & & & \\
\hline$\# 32$ & & & & & & & & \\
\hline$\# 33$ & & & & & & & & \\
\hline$\# 34$ & & & & & & & & \\
\hline$\# 35$ & & & & & & & & \\
\hline$\# 36$ & & & & & & & & \\
\hline$\# 37$ & & & & & & & & \\
\hline$\# 38$ & & & & & & & & \\
\hline$\# 39$ & & & & & & & & \\
\hline$\# 40$ & & & & & & & & \\
\hline$\# 41$ & & & & & & & & \\
\hline$\# 42$ & & & & & & & & \\
\hline$\# 43$ & & & & & & & & \\
\hline$\# 44$ & & & & & & & & \\
\hline$\# 45$ & & & & & & & & \\
\hline$\# 46$ & & & & & & & & \\
\hline$\# 47$ & & & & & & & & \\
\hline$\# 48$ & & & & & & & & \\
\hline$\# 49$ & & & & & & & & \\
\hline$\# 50$ & & & & & & & & \\
\hline$\# 51$ & & & & & & & & \\
\hline$\# 52$ & & & & & & & & \\
\hline$\# 53$ & & & & & & & & \\
\hline$\# 54$ & & & & & & & & \\
\hline$\# 55$ & & & & & & & & \\
\hline$\# 56$ & & & & & & & & \\
\hline$\# 57$ & & & & & & & & \\
\hline$\# 58$ & & & & & & & & \\
\hline$\# 59$ & & & & & & & & \\
\hline$\# 60$ & & & & & & & & \\
\hline$\# 61$ & & & & & & & & \\
\hline$\# 62$ & & & & & & & & \\
\hline$\# 63$ & & & & & & & & \\
\hline
\end{tabular}


explore their available database and verify the prevalence of MRI findings or clinical correlates in the whole or in specific populations after repeated exposure to GBCAs.

While radiologists consider providing diagnostic imaging with contrast-enhanced MRI a professional and ethical obligation, there is an equal obligation to protect patients from the potential risk of side effects related to this diagnostic procedure.

The current guidelines adopted by regulatory agencies (EMA, European Medicine Agency; FDA, Food and Drug Administration) and scientific societies (ESUR, European Society of Urogenital Radiology; ACR, American College of Radiology etc.) are relevant and of great importance in the clinical management of patients with renal insufficiency. However, methodological aspects are crucial to harmonize data and support decision-making both at preclinical [65] and clinical level [66]. In fact, recommendations on how to design and report data from clinical studies on gadoliniuminduced brain $\mathrm{T} 1$ hyperintensity are currently missing.

\section{Evidence from preclinical research}

The appearance of T1w hyperintensity in the dentate nucleus of the cerebellum and in other brain areas is under debate as it has been used as an MRI correlate of gadolinium deposition in the brain and to compare different GBCAs. The data available from the scientific literature may appear not consistent among MRI studies; the retrospective nature of the studies and variables depending on patient's history, types of GBCAs used (confounded vs. unconfounded), and MRI protocols affect the results.

The currently prevailing theory of the development of gadolinium-dependent $\mathrm{T} 1 \mathrm{w}$ hyperintensity is based on animal studies that found gadolinium in three different forms: the intact chelate, precipitated gadolinium, and gadolinium bound to macromolecules $[65,67,68]$. While the intact chelate was found for both - linear and macrocyclic chelates - precipitated gadolinium or gadolinium bound to macromolecules was found exclusively for linear GBCAs [69]. A recent study reported a washout of the intact chelate for the macrocyclic GBCA gadoterate (with no gadolinium above the level of detection after 6 months) while pharmacokinetic analysis suggested that gadolinium bound to macromolecules might stay permanently in the brain $[68,70]$. Since, at least at the magnetic fields used in clinical practice, precipitated gadolinium should not cause T1-shortening and gadolinium bound to macromolecules causes a strong T1-effect, it is very likely that gadolinium released from the less stable linear agents that subsequently binds to macromolecules is the cause of the observed T1w hyperintensity seen with linear GBCAs. The accumulation of gadolinium in the DN and other structures might further be explained by the increased metal content in these areas, facilitating transmetallation.

In contrast, the intact chelate might only cause a temporary signal intensity increase during the washout phase of the chelate. The pathway of the intact complex through the brain is not completely understood, but there is accumulating evidence that gadolinium gets into the CSF through the choroid plexus and reaches the brain through the glymphatic system. It is important to point out that animal studies provided evidence that a comparable amount of all GBCAs reaches the brain and might cause $\mathrm{T} 1 \mathrm{w}$ hyperintensities on its way through the glymphatic system $[68,71]$. However, T1w hyperintensities in metal-rich areas should exclusively appear if the gadolinium is released from its complex. Hence, it is not expected that the intact chelate will accumulate in the DN or other metalrich structures. These considerations underline the importance of the appearance of a visible dentate hyperintensity on unenhanced $\mathrm{T} 1 \mathrm{w}$ images and do not give a theoretical basis for a long-lasting signal intensity increase (that is not caused by the temporary presence of the intact chelate) if no gadolinium accumulation can be found.

It should be emphasized that the T1w signal intensity increase in the brain might represent an indicator of the gadolinium release in the whole body. Especially the bone and skin [72-74] have been identified as areas of gadolinium deposition.

In summary, multiple biochemical and physiological mechanisms can modulate relaxivities in the living tissues such as macromolecular binding, water access, aggregation/precipitation, and cellular internalization. These effects are complex and do not affect $\mathrm{T} 1, \mathrm{~T} 2$, and $\mathrm{T} 2 *$ relaxivity the same way. Thus, a fraction
Table 1 Type I and Type II Errors in Data Interpretation of Signal Intensity of the Dentate Nucleus and other brain sites after repetitive administrations of GBCAs

\begin{tabular}{ll}
\hline Type I errors & Type II errors \\
\hline Disease mechanisms & MRI Technical aspects (e.g., GRE vs. SE sequences) \\
Age effects & Age effects \\
Confounded GBCAs (e.g., exposure to linear GBCAs & Confounded GBCAs (e.g., exposure to macrocyclic \\
in studies focused on macrocyclic GBCAs) & GBCAs in studies focused on linear GBCAs) \\
Presence of other minerals (e.g., $\mathrm{Ca}^{2+}, \mathrm{Fe}^{3+}, \mathrm{Cu}^{3+}, \mathrm{Mn}^{2+}$ ) & $\mathrm{Fe}^{3+}$ effects on SI on T1 weighted images \\
Macromolecular complexes with high $\mathrm{T} 1 \mathrm{relaxivity}$ & Lower T1 relaxivity at higher magnetic fields \\
& (e.g., 3.0 T vs. 1.5 T) \\
& Absence of signal from precipitated $\mathrm{Gd}^{3+}$ \\
& salts in tissue \\
\hline
\end{tabular}


of the deposited gadolinium will remain invisible on T1w images. Multiparametric MRI analyses (e.g., with combination of T1, T2, and $\mathrm{T} 2 *$ or quantitative magnetic susceptibility mapping) may be used in future studies to disentangle these combined effects.

\section{Recommendations}

Results of the analysis of current literature indicate that knowledge gaps exist and recommendations are needed to systematically collect data in the experimental design of scientific studies [64, 75]. In November 2016 and 2017, the 1st and 2nd European Gadolinium Retention Evaluation Consortium (GREC) meetings were held in Naples (Italy) and Lisbon (Portugal), respectively, under the patronage of the European Society of Neuroradiology, with the intent to join the efforts of scientists from academy and industry and improve the understanding of the mechanisms of gadolinium retention/deposition/accumulation in the brain and non-brain tissues and, if any, its relationship with clinical symptoms in patients exposed to GBCAs [64]. In 2016, the GREC commissioned a Task Force to review the evidence regarding the gadolinium-dependent $\mathrm{T} 1 \mathrm{~W}$ hyperintensity in the brain and the methodology used to assess gadolinium retention in the brain in clinical studies.

In developing this position statement, the GREC and its Task Force assessed the methods applied to the measurement of T1w high SI in the brain in retrospective studies and found a lack of consistency in methodology across different institutions.

National and international scientific societies may use this recommendation to solicit and design harmonized multicenter studies that are aimed to explore indirect MRI measures of gadolinium retention/deposition in the brain. Regulatory agencies and scientific journal editors could use these recommendations to verify the methodology used in clinical studies. Finally, scientists will find a reference and a tool for the design and development of clinical studies.

\section{Recommendations for the study design and interpretation of MRI results in clinical studies}

Since restriction policies on the use of contrast agents must be evidence-based and excessive restrictions can have negative implications for healthcare, the GREC Task Force proposes the following specific suggestions (summarized in Table 2) to conduct clinical studies that assess brain MRI changes following repetitive intravenous administrations of GBCAs and to increase data homogeneity among different institutions.

1. GBCA administration has to be registered and traced over time to allow comparison of gadolinium retentiondependent signal. Institutions must have unequivocal proof of the type and dose of GBCA administered in each examination. If an institution has shifted from the single use of one GBCA compound to the single use of another one, shift dates should be clearly defined and documented. Authors have to exclude exposure to other GBCAs in the assessed period unequivocally by chart review. If they cannot exclude exposure to other GBCAs in the assessed period of time, they clearly have to state this.

2. Only SI differences between two specific MRI studies or intra-individual serial evaluation of multiple MRI studies should be assessed. Since absolute SIs are not reliable to assess gadolinium retention based on the influence of many parameters such as coil sensitivity, coil filling factor, head positioning within the coil, coil tuning/ matching drift, MRI parameter drift, hardware and software gain, only relative SIs should be assessed. If available, a control group which got either a different GBCA or no GBCAs at all might be assessed.

3. Since the relevant parameter is the SI change, patients with pre-existing hyperintensity due to the prior injection of linear GBCAs can be included even if they display T1w high signal intensity at baseline. However, the authors need to provide documentation or state that they cannot exclude that the patients received other GBCAs prior to the inclusion in the study. A subgroup analysis may need to be conducted to exclude influence of previous GBCA injections or other confounding disease processes.

4. The average number of injections and the time between injections need to be documented clearly.

5. The DN has been shown to be the most sensitive structure for gadolinium deposition. Thus, studies that assess gadolinium retention in the brain should focus on this structure. If no SI increase is found in the DN, it is unnecessary to assess any further structure in the brain. If an SI increase in the DN is found, further structures (such as the GP) might be assessed.

6. The DN-to-pons ratio should be assessed in all studies since this is the most often used ratio in previous studies. Additionally, the DN-to-middle cerebellar peduncle (MCP) ratio can be used. The reliability of these measures is mainly based on the observation of less gadolinium deposition in the pons and middle cerebellar peduncles compared to DN in autopsy studies.

7. Regions of interest (ROI)-based approaches have been the most used and are expected to be used in future retrospective analyses. Generally, an extremely low inter-rater variability of this approach has been reported in previous studies if conducted appropriately. Since the DN is better depicted on T2w or diffusion-weighted images, these should be used as reference for placing ROIs. Since ROI drawing remains operator-dependent and used to obtain relative SI ratios, intra-rater and inter-rater agreement might be conducted as internal quality check. 
Table 2 Recommendations to design retrospective studies on changes of signal intensity on unenhanced T1-weighted images after repetitive intravenous administrations of GBCAs

\begin{tabular}{ll}
\hline Institution & - Provide unequivocal proof of the type and dose of GBCA \\
used in each examination. \\
Patients
\end{tabular}

GBCA gadolinium-based contrast agent, SI signal intensity
8. Performance of automatic segmentation techniques in comparison to ROI techniques might be considered. Due to the low reported inter-rater variability of the ROI-based approach, it is questionable if the automatic approaches might provide additional value. However, this approach could be useful for the assessment of large datasets and of studies that provided unexpected results (e.g., high SI increase without any visible hyperintensity or no SI changes with visible hyperintensity).

9. Quantitative analyses such as T1 and T2 relaxivity mapping may be used additionally. It is currently unclear if they can provide additional value.

10. Since several technical parameters can affect the MR signal, investigators should indicate in the methods section a detailed table with the MR parameters: B0 static magnetic field, MR magnet vendor, coil type and number of coil channels, gradient strength, type of pulse sequence (e.g., Spin Echo (SE), Fast/Turbo Spin Echo (FSE or TSE), Gradient Echo (GRE), Inversion Recovery (IR), Magnetization Prepared Gradient Echo (MP-RAGE), type of prepulse, type of phase encoding (2D or 3D), Fourier transformation algorithm, repetition time (TR), echo time (TE), inversion time if applicable, flip angle, slice thickness, voxel volume, field of view, matrix, number of averages, and receiver bandwidth.

11. The SI ratio difference can only be calculated between MRI examinations that use identical imaging parameters and field strengths. Variation for TR and TE values between exams should be kept as small as possible. The maximum threshold of $3.5 \%$ of variation has been proposed as acceptable [24]. For high levels of parameter variations, subgroup analyses need to be performed. Variations of TR and TE above $15 \%$ should be excluded [24]. The SI ratio difference cannot be calculated between different pulse sequences or magnetic field strengths.

12. All studies should check if there is a visible SI increase in the DN in all patients that cannot be explained by confounders. Studies that report a high SI increase without corresponding visibility of this increase might be flawed (e.g., measurement of aging-related changes). The same holds true for studies that find clear visible SI increase in some patients but did not find a statistically significant SI increase.

13. Even though no clear correlation between SI increase and symptoms has been shown for most diseases, as a general recommendation, it is appropriate to evaluate and compare groups of patients with similar diagnoses. The available data do not allow understanding, at this point, of what is the contribution of the underlying pathologic process (brain tumors, chronic inflammatory diseases) on gadolinium retention and deposition. In addition, pediatric patients should always be treated as a distinct group for analysis.

14. Age of the patients included into retrospective studies should always be reported. This is crucial especially in pediatric patients that should be treated as a distinct group for analysis. 
15. In studies that report a SI increase, it is mandatory to consider the confounders of conditions that increase the concentration of manganese in the deep gray matter (parenteral feeding, exposure to manganese, portosystemic shunt) or certain disorders associated with an increase of the SI of the basal ganglia (neurofibromatosis type I, metabolic disorders, hypoxic-ischemic encephalopathy, etc.). The influence of radiation as a confounder is still a topic under investigation [52].

\section{Conclusions}

There is a broad consensus that radiologists should use GBCAs with the minimum effective dosage and only when strictly clinically indicated. At the same time, every effort should be made to protect patients from any side effect related to retention or exposure to GBCAs, and concern for public safety is paramount, particularly in children and patients who need several follow-up exams.

It must be underlined that there is currently no evidence of clinical consequences after exposure and retention of gadolinium in brain and non-brain tissues of patients with normal renal function, but any potential gadolinium-induced toxicity should also not be underestimated.

The current recommendations are a joint international effort (GREC) to increase the comparability of the huge number of retrospective studies that are currently published on SI increase on T1w MRI following serial injections of GBCAs. We encourage authors to follow these recommendations and editors and reviewers to evaluate the methodology of the papers submitted for publication to peer-reviewed journals as there is a need of data homogeneity and standardization on this topic.

Funding The GREC Task Force is an independent, voluntary body. No funding supported this work.

\section{Compliance with ethical standards}

Guarantor The scientific guarantor of this publication is Carlo C. Quattrocchi, Departmental Faculty of Medicine and Surgery, Head of the Unit of Diagnostic Imaging and Interventional Radiology, Università Campus Bio-Medico di Roma, Rome, Italy.

Conflict of interest The authors of this manuscript declare relationships with the following companies:

C.C. Quattrocchi has received speaker honoraria from Bayer Healthcare; has organized the 1st and 2nd European GREC meetings in 2016 and 2017 sponsored by Bayer, Bracco, GE, and Guerbet.

J. Ramalho has organized the 2nd European GREC meeting sponsored by Bayer, Bracco, GE and Guerbet.

A. J. van der Molen has received chairman honoraria from Guerbet; has organized the 1st and 2nd European GREC meetings in 2016 and 2017 sponsored by Bayer, Bracco, GE, and Guerbet.
À. Rovira serves on scientific advisory boards for Novartis, SanofiGenzyme, Icometrix, SyntheticMR, and OLEA Medical, and has received speaker honoraria from Bayer Healthcare, Sanofi-Genzyme, Bracco, Merck-Serono, Teva Pharmaceutical Industries Ltd., Novartis, Roche, and Biogen Idec.

A. Radbruch: Bayer (invited talks, study funding, consultancy, advisory boards), Bracco (advisory board), Guerbet (invited talks, study funding, consultancy), GE (advisory board).

Statistics and biometry No complex statistical methods were necessary for this paper.

Informed consent Written informed consent was not required for this study because no original data were produced.

Ethical approval Institutional Review Board approval was not required because the paper is a recommendation statement and experiments were not performed.

Methodology International consensus statement on methodological recommendations on clinical studies

\section{References}

1. Kanda T, Ishii K, Kawaguchi H, Kitajima K, Takenaka D (2014) High signal intensity in the dentate nucleus and globus pallidus on unenhanced T1-weighted MR images: relationship with increasing cumulative dose of a gadolinium-based contrast material. Radiology 270:834-841

2. Errante Y, Cirimele V, Mallio CA, Di Lazzaro V, Zobel BB, Quattrocchi CC (2014) Progressive increase of T1 signal intensity of the dentate nucleus on unenhanced magnetic resonance images is associated with cumulative doses of intravenously administered gadodiamide in patients with normal renal function, suggesting dechelation. Invest Radiol 49:685-690

3. Kanda T, Osawa M, Oba $\mathrm{H}$ et al (2015) High signal intensity in dentate nucleus on unenhanced T1-weighted MR images: association with linear versus macrocyclic gadolinium chelate administration. Radiology 275:803-809

4. Quattrocchi CC, Mallio CA, Errante Y et al (2015) Gadodiamide and dentate nucleus T1 Hyperintensity in patients with meningioma evaluated by multiple follow-up contrast-enhanced magnetic resonance examinations with no systemic interval therapy. Invest Radiol 50:470 472

5. Radbruch A, Weberling LD, Kieslich PJ et al (2015) Gadolinium retention in the dentate nucleus and globus pallidus is dependent on the class of contrast agent. Radiology 275:783-791

6. Miller JH, Hu HH, Pokorney A, Cornejo P, Towbin R (2015) MRI brain signal intensity changes of a child during the course of 35 gadolinium contrast examinations. Pediatrics 136:e1637-e1640

7. Ramalho J, Castillo M, AlObaidy M et al (2015) High signal intensity in globus pallidus and dentate nucleus on unenhanced T1weighted MR images: evaluation of two linear gadolinium-based contrast agents. Radiology 276:836-844

8. Stojanov DA, Aracki-Trenkic A, Vojinovic S, Benedeto-Stojanov D, Ljubisavljevic S (2016) Increasing signal intensity within the dentate nucleus and globus pallidus on unenhanced T1W magnetic resonance images in patients with relapsing-remitting multiple sclerosis: correlation with cumulative dose of a macrocyclic gadolinium-based contrast agent, gadobutrol. Eur Radiol 26:807815

9. Adin ME, Kleinberg L, Vaidya D, Zan E, Mirbagheri S, Yousem DM (2015) Hyperintense dentate nuclei on T1-weighted MRI: 
relation to repeat gadolinium administration. AJNR Am J Neuroradiol 36:1859-1865

10. McDonald RJ, McDonald JS, Kallmes DF et al (2015) Intracranial gadolinium deposition after contrast-enhanced MR imaging. Radiology 275:772-782

11. Weberling LD, Kieslich PJ, Kickingereder P et al (2015) Increased signal intensity in the dentate nucleus on unenhanced T1-weighted images after gadobenate dimeglumine administration. Invest Radiol 50:743-748

12. Radbruch A, Weberling LD, Kieslich PJ et al (2015) High-signal intensity in the dentate nucleus and Globus pallidus on unenhanced T1-weighted images: evaluation of the macrocyclic gadoliniumbased contrast agent gadobutrol. Invest Radiol 50:805-810

13. Cao Y, Huang DQ, Shih G, Prince MR (2016) Signal change in the dentate nucleus on T1-weighted MR images after multiple administrations of gadopentetate dimeglumine versus gadobutrol. AJR Am J Roentgenol 206:414-419

14. Ramalho J, Semelka RC, AlObaidy M, Ramalho M, Nunes RH, Castillo M (2016) Signal intensity change on unenhanced T1weighted images in dentate nucleus following gadobenate dimeglumine in patients with and without previous multiple administrations of gadodiamide. Eur Radiol 26:4080-4088

15. Ramalho J, Ramalho M, AlObaidy M, Nunes RH, Castillo M, Semelka RC (2016) T1 signal-intensity increase in the dentate nucleus after multiple exposures to gadodiamide: intraindividual comparison between 2 commonly used sequences. AJNR Am J Neuroradiol 37(8):1427-1431

16. Tedeschi E, Palma G, Canna A et al (2016) In vivo dentate nucleus MRI relaxometry correlates with previous administration of gadolinium-based contrast agents. Eur Radiol 26(12):4577-4584

17. Roberts DR, Chatterjee AR, Yazdani M et al (2016) Pediatric patients demonstrate progressive T1-weighted hyperintensity in the dentate nucleus following multiple doses of gadolinium-based contrast agent. AJNR Am J Neuroradiol 37(12):2340-2347

18. Tanaka M, Nakahara K, Kinoshita M (2016) Increased signal intensity in the dentate nucleus of patients with multiple sclerosis in comparison with neuromyelitis optica spectrum disorder after multiple doses of gadolinium contrast. Eur Neurol 75:195-198

19. Cao Y, Zhang Y, Shih G et al (2016) Effect of renal function on gadolinium-related signal increases on unenhanced T1-weighted brain magnetic resonance imaging. Invest Radiol 51:677-682

20. Hu HH, Pokorney A, Towbin RB, Miller JH (2016) Increased signal intensities in the dentate nucleus and globus pallidus on unenhanced T1-weighted images: evidence in children undergoing multiple gadolinium MRI exams. Pediatr Radiol 46:1590-1598

21. Roberts DR, Holden KR (2016) Progressive increase of T1 signal intensity in the dentate nucleus and globus pallidus on unenhanced T1-weighted MR images in the pediatric brain exposed to multiple doses of gadolinium contrast. Brain Dev 38:331-336

22. Khant ZA, Hirai T, Kadota Y et al (2017) T1 shortening in the cerebral cortex after multiple administrations of gadolinium-based contrast agents. Magn Reson Med Sci 16:84-86

23. Eisele P, Alonso A, Szabo K et al (2016) Lack of increased signal intensity in the dentate nucleus after repeated administration of a macrocyclic contrast agent in multiple sclerosis: an observational study. Medicine (Baltimore) 95:e4624

24. Radbruch A, Weberling LD, Kieslich PJ et al (2016) Intraindividual analysis of signal intensity changes in the dentate nucleus after consecutive serial applications of linear and macrocyclic gadolinium-based contrast agents. Invest Radiol 51:683-690

25. Zhang Y, Cao Y, Shih GL, Hecht EM, Prince MR (2017) Extent of signal hyperintensity on unenhanced T1-weighted brain MR images after more than 35 administrations of linear gadoliniumbased contrast agents. Radiology 282:516-525

26. Eisele P, Szabo K, Alonso A et al (2018) Lack of T1 hyperintensity in the dentate nucleus after 15 administrations of a macrocyclic contrast agent in multiple sclerosis. J Neurol Neurosurg Psychiatry 89:324-326

27. Schlemm L, Chien C, Bellmann-Strobl J et al (2017) Gadopentetate but not gadobutrol accumulates in the dentate nucleus of multiple sclerosis patients. Mult Scler 23:963-972

28. Radbruch A, Haase R, Kieslich PJ et al (2017) No signal intensity increase in the dentate nucleus on unenhanced T1-weighted MR images after more than 20 serial injections of macrocyclic gadolinium-based contrast agents. Radiology 282:699-707

29. Kuno H, Jara H, Buch K, Qureshi MM, Chapman MN, Sakai O (2017) Global and regional brain assessment with quantitative MR imaging in patients with prior exposure to linear gadolinium-based contrast agents. Radiology 283:195-204

30. Bae S, Lee HJ, Han K et al (2017) Gadolinium deposition in the brain: association with various GBCAs using a generalized additive model. Eur Radiol 27:3353-3361

31. Radbruch A, Haase R, Kickingereder P et al (2017) Pediatric brain: no increased signal intensity in the dentate nucleus on unenhanced T1-weighted MR images after consecutive exposure to a macrocyclic gadolinium-based contrast agent. Radiology 283(3):828-836

32. Flood TF, Stence NV, Maloney JA, Mirsky DM (2017) Pediatric brain: repeated exposure to linear gadolinium-based contrast material is associated with increased signal intensity at unenhanced T1weighted MR imaging. Radiology 282:222-228

33. Langner S, Kromrey ML, Kuehn JP, Grothe M, Domin M (2017) Repeated intravenous administration of gadobutrol does not lead to increased signal intensity on unenhanced T1-weighted images-a voxel-based whole brain analysis. Eur Radiol 27:3687-3693

34. Kahn J, Posch H, Steffen IG et al (2017) Is there long-term signal intensity increase in the central nervous system on T1-weighted images after MR imaging with the hepatospecific contrast agent gadoxetic acid? A cross-sectional study in 91 patients. Radiology 282:708-716

35. Ichikawa S, Motosugi U, Omiya Y, Onishi H (2017) Contrast agentinduced high signal intensity in dentate nucleus on unenhanced T1weighted images: comparison of gadodiamide and gadoxetic acid. Invest Radiol 52:389-395

36. Conte G, Preda L, Cocorocchio E et al (2017) Signal intensity change on unenhanced T1-weighted images in dentate nucleus and globus pallidus after multiple administrations of gadoxetate disodium: an intraindividual comparative study. Eur Radiol 27:4372-4378

37. Tedeschi E, Cocozza S, Borrelli P, Ugga L, Morra VB, Palma G (2018) Longitudinal assessment of dentate nuclei relaxometry during massive gadobutrol exposure. Magn Reson Med Sci 17:100-104

38. Rossi Espagnet MC, Bernardi B, Pasquini L, Figà-Talamanca L, Tomà $\mathrm{P}$, Napolitano A (2017) Signal intensity at unenhanced T1weighted magnetic resonance in the globus pallidus and dentate nucleus after serial administrations of a macrocyclic gadoliniumbased contrast agent in children. Pediatr Radiol 47:1345-1352

39. Forslin Y, Shams S, Hashim F et al (2017) Retention of gadoliniumbased contrast agents in multiple sclerosis: retrospective analysis of an 18-year longitudinal study. AJNR Am J Neuroradiol 38:1311-1316

40. Roberts DR, Welsh CA, LeBel DP 2nd, Davis WC (2017) Distribution map of gadolinium deposition within the cerebellum following GBCA administration. Neurology 88:1206-1208

41. Schneider GK, Stroeder J, Roditi G et al (2017) T1 signal measurements in pediatric brain: findings after multiple exposures to gadobenate dimeglumine for imaging of nonneurologic disease. AJNR Am J Neuroradiol 38:1799-1806

42. Eisele P, Konstandin S, Szabo K et al (2017) Sodium MRI of T1 high signal intensity in the dentate nucleus due to gadolinium deposition in multiple sclerosis. J Neuroimaging 27:372-375

43. Tibussek D, Rademacher C, Caspers J et al (2017) Gadolinium brain deposition after macrocyclic gadolinium administration: a pediatric case-control study. Radiology 285:223-230

44. Splendiani A, Perri M, Marsecano C et al (2018) Effects of serial macrocyclic-based contrast materials gadoterate meglumine and 
gadobutrol administrations on gadolinium-related dentate nuclei signal increases in unenhanced T1-weighted brain: a retrospective study in 158 multiple sclerosis (MS) patients. Radiol Med 123:125-134

45. Lee JY, Park JE, Kim HS et al (2017) Up to 52 administrations of macrocyclic ionic MR contrast agent are not associated with intracranial gadolinium deposition: multifactorial analysis in 385 patients. PLoS One 12:e183916

46. Bjørnerud A, Vatnehol SAS, Larsson C, Due-Tønnessen P, Hol PK, Groote IR (2017) Signal enhancement of the dentate nucleus at unenhanced MR imaging after very high cumulative doses of the macrocyclic gadolinium-based contrast agent gadobutrol: an observational study. Radiology 285:434-444

47. Yoo RE, Sohn CH, Kang KM et al (2018) Evaluation of gadolinium retention after serial administrations of a macrocyclic gadoliniumbased contrast agent (gadobutrol): a single-institution experience with 189 patients. Invest Radiol 53:20-25

48. Müller A, Jurcoane A, Mädler B, Ditter P, Schild H, Hattingen E (2017) Brain relaxometry after macrocyclic Gd-based contrast agent. Clin Neuroradiol 27:459-468

49. Kromrey ML, Liedtke KR, Ittermann T et al (2017) Intravenous injection of gadobutrol in an epidemiological study group did not lead to a difference in relative signal intensities of certain brain structures after 5 years. Eur Radiol 27:772-777

50. Young JR, Orosz I, Franke MA et al (2018) Gadolinium deposition in the paediatric brain: T1-weighted hyperintensity within the dentate nucleus following repeated gadolinium-based contrast agent administration. Clin Radiol 73:290-295

51. Renz DM, Kümpel S, Böttcher J et al (2018) Comparison of unenhanced T1-weighted signal intensities within the dentate nucleus and the globus pallidus after serial applications of gadopentetate dimeglumine versus gadobutrol in a pediatric population. Invest Radiol 53:119-127

52. Tamrazi B, Nguyen B, Liu CJ et al (2018) Changes in signal intensity of the dentate nucleus and globus pallidus in pediatric patients: impact of brain irradiation and presence of primary brain tumors independent of linear gadolinium-based contrast agent administration. Radiology 287:452-460

53. Ryu YJ, Choi YH, Cheon JE et al (2018) Pediatric brain: gadolinium deposition in dentate nucleus and globus pallidus on unenhanced T1-weighted images is dependent on the type of contrast agent. Invest Radiol 53:246-255

54. Vergauwen E, Vanbinst AM, Brussaard C et al (2018) Central nervous system gadolinium accumulation in patients undergoing periodical contrast MRI screening for hereditary tumor syndromes. Hered Cancer Clin Pract 16:2

55. Moser FG, Watterson CT, Weiss $\mathrm{S}$ et al (2018) High signal intensity in the dentate nucleus and globus pallidus on unenhanced t1weighted $\mathrm{mr}$ images: comparison between gadobutrol and linear gadolinium-based contrast agents. AJNR Am J Neuroradiol. https://doi.org/10.3174/ajnr.A5538

56. Kasper E, Schemuth HP, Horry S, Kinner S (2018) Changes in signal intensity in the dentate nucleus at unenhanced T1-weighted magnetic resonance imaging depending on class of previously used gadolinium-based contrast agent. Pediatr Radiol 48:686-693

57. Kinner S, Schubert TB, Bruce RJ et al (2018) Deep brain nuclei T1 shortening after gadobenate dimeglumine in children: influence of radiation and chemotherapy. AJNR Am J Neuroradiol 39:24-30

58. Quattrocchi CC, Errante Y, Mallio CA et al (2018) Effect of age on high T1 signal intensity of the dentate nucleus and globus pallidus in a large population exposed to gadodiamide. Invest Radiol 53:214-222

59. Bolles GM, Yazdani M, Stalcup ST et al (2018) Development of high signal intensity within the globus pallidus and dentate nucleus following multiple administrations of gadobenate dimeglumine. AJNR Am J Neuroradiol. https://doi.org/10.3174/ajnr.A5510

60. Pei L, Xu J, Zhang M (2017) Correlation between high signal intensity in cerebrum nucleus on unenhanced T1-weighted MR images and number of previous gadolinium-based contrast agent administration. Zhejiang Da Xue Xue Bao Yi Xue Ban 46:487-491

61. Behzadi AH, Farooq Z, Zhao Y, Shih G, Prince MR (2018) Dentate nucleus signal intensity decrease on T1-weighted MR images after switching from gadopentetate dimeglumine to gadobutrol. Radiology 287:816-823

62. Young JR, Qiao J, Orosz I et al (2018) Gadolinium deposition within the paediatric brain: no increased intrinsic T1-weighted signal intensity within the dentate nucleus following the administration of a minimum of four doses of the macrocyclic agent gadobutrol. Eur Radiol. https://doi.org/10.1007/s00330-018-5464-5

63. Rahatli FK, Donmez FY, Kibaroglu S et al (2018) Does renal function affect gadolinium deposition in the brain? Eur J Radiol 104:33-37

64. Quattrocchi CC, van der Molen AJ (2017) Gadolinium retention in the body and brain: is it time for an international joint research effort? Radiology 282:12-16

65. Frenzel T, Apte C, Jost G, Schöckel L, Lohrke J, Pietsch H (2017) Quantification and assessment of the chemical form of residual gadolinium in the brain after repeated administration of gadolinium-based contrast agents: comparative study in rats. Invest Radiol 52:396-404

66. Dekkers IA, Roos R, van der Molen AJ (2018) Gadolinium retention after administration of contrast agents based on linear chelators and the recommendations of the European Medicines Agency. Eur Radiol 28:1579-1584

67. Gianolio E, Bardini P, Arena F et al (2017) Gadolinium retention in the rat brain: assessment of the amounts of insoluble gadoliniumcontaining species and intact gadolinium complexes after repeated administration of gadolinium-based contrast agents. Radiology 285:839-849

68. Robert P, Fingerhut S, Factor C et al (2018) One-year retention of gadolinium in the brain: comparison of gadodiamide and gadoterate meglumine in a rodent model. Radiology 288(2):424-433

69. Radbruch A, Roberts DR, Clement O, Rovira A, Quattrocchi CC (2017) Chelated or dechelated gadolinium deposition. Lancet Neurol 16:955

70. Radbruch A (2018) Gadolinium deposition in the brain: we need to differentiate between chelated and dechelated gadolinium. Radiology 88(2):434-435

71. Lohrke J, Frisk AL, Frenzel T et al (2017) Histology and gadolinium distribution in the rodent brain after the administration of cumulative high doses of linear and macrocyclic gadolinium-based contrast agents. Invest Radiol 52(6):324-333

72. Gibby WA, Gibby KA, Gibby WA (2004) Comparison of Gd DTPA-BMA (Omniscan) versus Gd HP-DO3A (ProHance) retention in human bone tissue by inductively coupled plasma atomic emission spectroscopy. Invest Radiol 39:138-142

73. Tweedle MF, Wedeking P, Kumar K (1995) Biodistribution of radiolabeled, formulated gadopentetate, gadoteridol, gadoterate, and gadodiamide in mice and rats. Invest Radiol 30:372-380

74. Pietsch H, Lengsfeld P, Jost G, Frenzel T, Hütter J, Sieber MA (2009) Long-term retention of gadolinium in the skin of rodents following the administration of gadolinium-based contrast agents. Eur Radiol 19:1417-1424

75. Robert P, Frenzel T, Factor C et al (2018) Methodological aspects for preclinical evaluation of gadolinium presence in brain tissue: critical appraisal and suggestions for harmonization-a joint initiative. Invest Radiol 53:499-517 